\title{
Catadioptric system optimisation for omnidirectional Robocup MSL robots
}

\author{
Gil Lopes, Fernando Ribeiro \\ Industrial Electronics Department, Univ. of Minho, \\ Campus de Azurém, 4800-058 Guimarães, Portugal \\ gil@dei.uminho.pt, fernando@,dei.uminho.pt
}

\begin{abstract}
Omnidirectional Robocup MSL robots often use catadioptric vision systems in order to enable $360^{\circ}$ of field view. It comprises an upright camera facing a convex mirror, commonly spherical, parabolic or hyperbolic, that reflects the entire space around the robot. This technique is being used for more than a decade and in a similar way by most teams. Teams upgrade their cameras in order to obtain more and better information of the captured area in pixel quantity and quality, but a large image area outside the convex mirror is black and unusable. The same happens on the image centre where the robot shows itself. Some efficiency though, can be improved in this technique by the methods presented in this paper such as developing a new convex mirror and by repositioning the camera viewpoint. Using $3 \mathrm{D}$ modelling $\mathrm{CAD} / \mathrm{CAM}$ software for the simulation and $\mathrm{CNC}$ lathe mirror construction, some results are presented and discussed.
\end{abstract}

Keywords: Omnidirectional robots, Robocup MSL, catadioptric system, 3D modelling

\section{Introduction}

RoboCup Middle Size league (MSL) robots are able to play soccer games autonomously. It is the major league of the whole RoboCup event. The robot's artificial vision system recognises the surrounding environment, the game field, the ball, the opponents and other obstacles by means of a catadioptric vision system in most participanting teams. Catadioptric or omnidirectional vision is known in MSL since late nineties when it was introduced for the MSL robots and literature about this technique is widely available. Fig. 1 shows the images captured by robot vision systems of three different MSL teams.
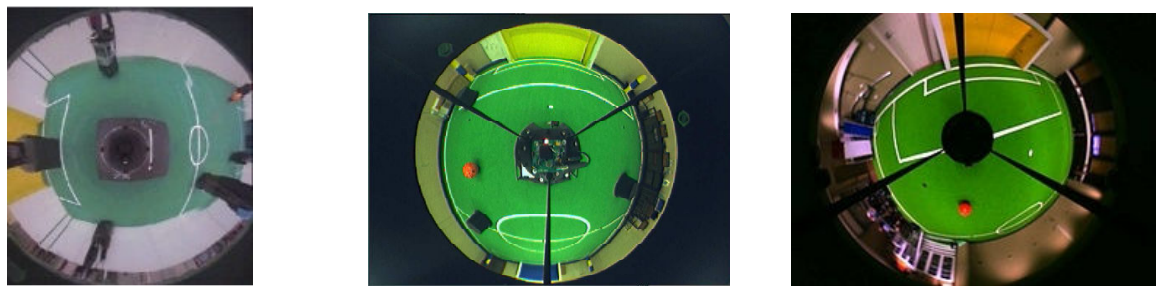

Fig. 1- Three examples of images captured by a catadioptric vision system from different MSL teams. Left - MRT team [1], Centre - Cambada [2], Right Brainstormers Tribots [3] 
Although this system is producing good results, its efficiency can be increased in order to extract more and better information from the captured image. It is important to extract as much as possible the necessary information from a captured image in order to process it flawlessly. This paper describes one approach divided in two methods to increase the efficiency of the catadioptric vision system. The paper is organised as follows. Section 2 briefly describes how a catadioptric system for MSL robots works, section 3 describes how this work customises a convex mirror using 3D modelling and simulation, section 4 describes our MSL robot head construction and section 5 draws some conclusions.

\section{Catadioptric vision system for MSL robots}

A catadioptric vision system applied to MSL robots is basically an upright camera facing a convex mirror as it is shown in Fig. 2-a. This makes the robot head. The higher the mirror position, the farther the robot can see. The head's position in relation to the robot can be seen in Fig. 2-b. Fig. 2-c shows a 3D computer model of the robot obtained from CAD software where the whole robot was drawn before being built. Using the same CAD technology, further in this paper it will be shown how a convex mirror was developed and simulated.

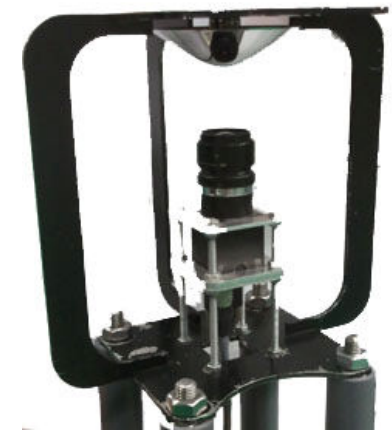

(a)

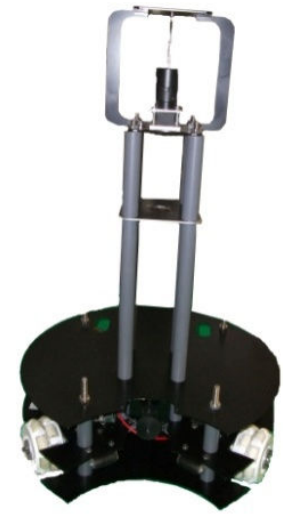

(b)

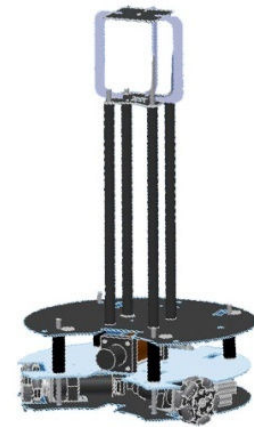

(c)

Fig. 2 - (a) Minho MSL robot head comprising an upright camera facing a convex mirror. (b) Picture of Minho MSL robot and respective head position. (c) 3D computer model of the robot from CAD software

\subsection{Types of convex mirrors}

Convex mirrors can be of different types such as conical, spherical, cylindrical, paraboloidal, ellipsoidal and hyperboloidal. Commonly applied to vision systems are spherical [4, 5] and hyperboloidal [6, 7] convex mirrors, and some work was also found on multi-part mirror construction made with a conical, spherical and planar parts [8]. A comparison of a spherical and hyperboloidal mirror types can also be found in [9]. Ishiguro has also reported the use of convex mirrors as it has compared different mirror shapes and their differences [10]. 


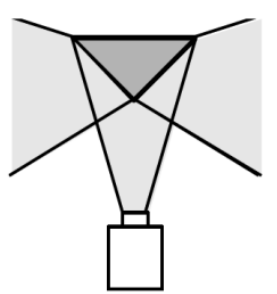

(a) Conic mirror

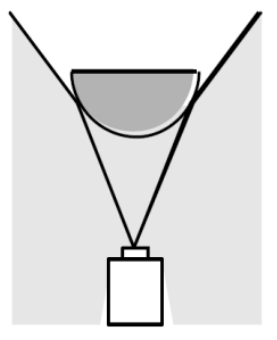

(b) Spherical mirror

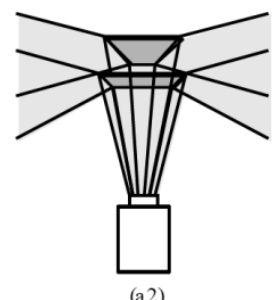

(a2)

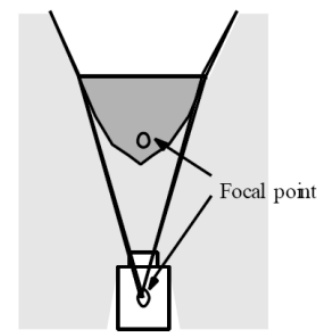

(c) Hyperboloidal mirror

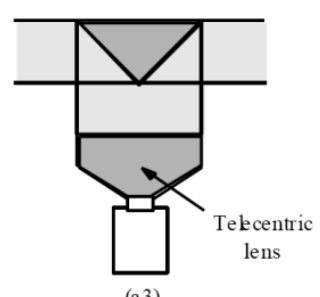

(a3)

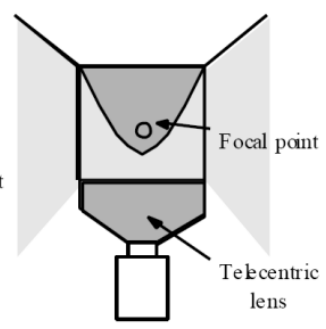

(d) Parabol h mir $\alpha$

Fig. 3 - Different omnidirectional mirrors [10]

The characterisation of a convex mirror and its shape can be performed mathematically; by trial and error or by $3 \mathrm{D}$ computer simulation. The first two ways are commonly found in the literature and the third way was the method used and described in this paper.

\section{Customising a convex mirror shape}

The sight of an omnidirectional vision system based on convex mirrors for MSL robots can be improved if the mirror is customised to the needs. In other words, a proper shape of the convex mirror can improve the robot's sight and therefore, improving its game playing. An empirical method based on 3D modelling is here proposed where the user simulates potential mirror shapes in order to obtain the desired image. After the shape is achieved the next step consists of exporting the data to a milling machine in order to build it. This is based on translating the data to a Computer Numerical Control (CNC) code as it is a direct approach from simulation to the real scenario. This work was carried out using a commercially available 3D modelling package (SolidWorks 2009 (C) [11]) and a CNC code (G-code) generation package (SolidCAM 2008 C) [12]).

\subsection{Computer 3D simulation}

A 3D model was developed based on the real size of the game field, ball and robots. The render option of SolidWorks allows the visualisation of the model in shading mode becoming a realistic view of the scene. Objects are rendered with their textures and when the parameters are set to obtain a mirror texture, the rendered image creates a texture in the object as a result of a reflection of the object's surroundings. This allows a more realistic simulation of a mirror object independently of its shape or form. 
The texture for the game field was developed to create a checkerboard type in one half of the field with a square side measuring $0.5 \mathrm{~m}$. In this way the distortion produced by the convex mirror can be assessed, measured and analysed directly. The other half is green and with the white field lines visible to simulate the real game field. Fig. 4-a shows an image of the simulated game field with some robots and game balls. SolidWorks also allows the simulation of a camera where the user can select the lens parameters. The rendered image can be generated from the camera perspective. The lens parameters were configured to match the lens used in our MSL robots and the camera was positioned at the same height and facing upwards to an object that simulated the convex mirror. The camera/lens and the object are the head of the robot, as shown in Fig. 4-b.

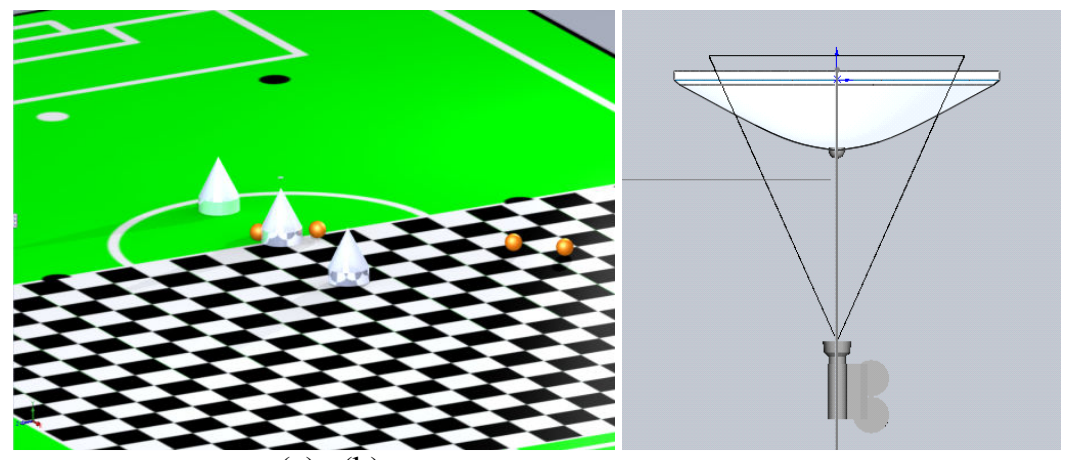

(a) (b)

Fig. 4 - (a) Computer simulated game field with robots and game balls and half of the game field with a checkerboard texture. (b) Simulation of the robot head with a camera/lens and the convex mirror object.

With this setup created, the work was then centred on the development of the mirror profile. This profile is made up of separate parts. A flat circular plate simulates the top of the convex mirror where the fixture is positioned. The mirror itself is based on a line that starts from the mirror centre and moves up to the sides. This line defines the mirror curvature and can be defined with continuous segments of a line each one performing different curvatures. A method called "revolve" from SolidWorks is then applied to the line to perform a $360^{\circ}$ turn around a point and in this case, the point is the mirror centre. By selecting a metallic texture with the shininess parameter to its maximum and applying it to the object, a mirror is created. All the reflection surface calculation is then left to the software. Fig. 5-a shows the shaped line made of three segments before the application of SolidWorks 'revolve' method and Fig. 5-b shows the final mirror aspect after applying SolidWorks 'revolve' method. The simulation is then applied by rendering or shading the final image from the point of view of the positioned camera. Fig. 6 shows rendered images of different convex mirror shapes and it is clear the differences of the surrounding object positions in relation to the image centre where the robot and camera are. Changing the line shape that defines the mirror profile allows the creation of any type of convex mirror and also allows the user to experience the resulting visualisation, in a few seconds. This technique also allows the creation of multi-part type mirrors as it was used to produce our actual developed mirror as explained next. 

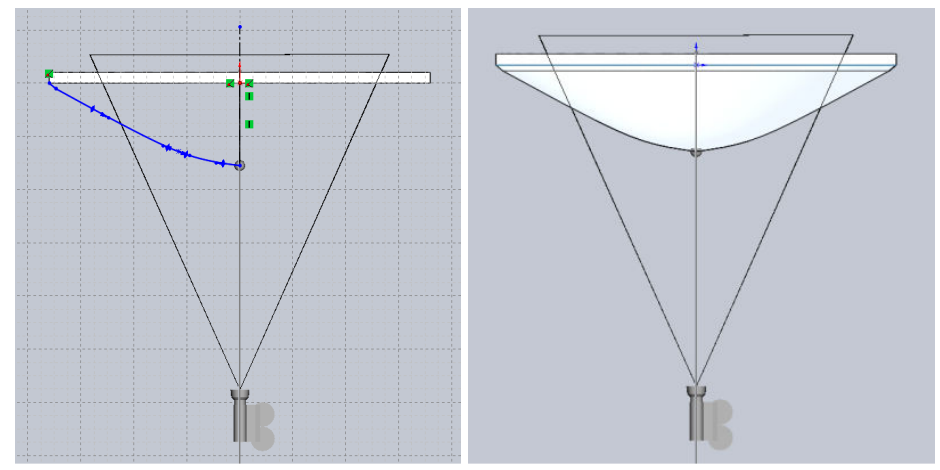

(a) (b)

Fig. 5 - (a) Convex mirror object with the shaped line before SolidWorks 'revolve' method. (b) Convex mirror object after the application of SolidWorks 'revolve' method
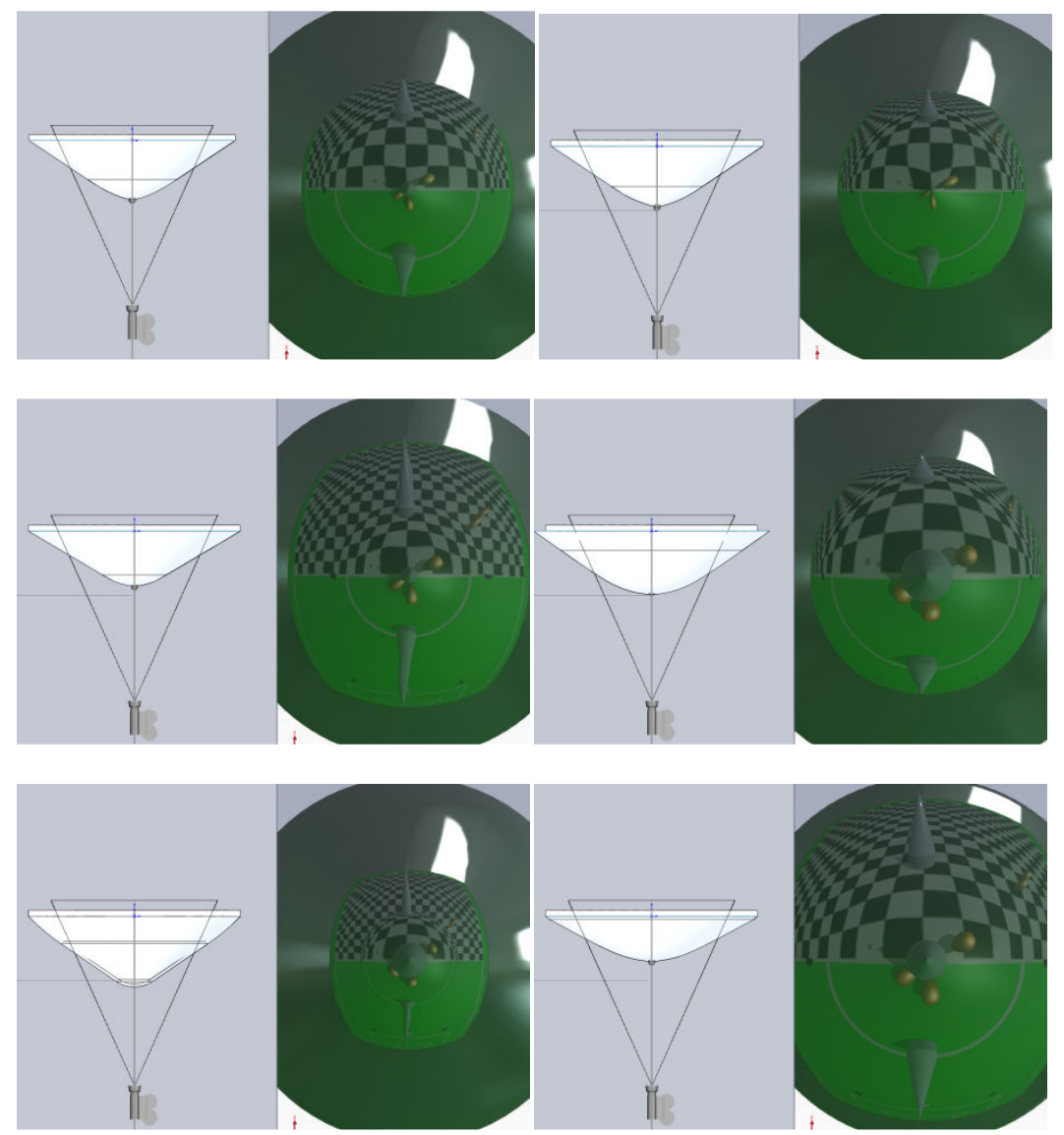

Fig. 6 - Rendered images of different convex mirror shapes 


\subsection{Simulated mirrors}

Our main goal was to create a multi-part convex mirror that could reflect the robot surroundings in different ways at defined radius. By examining the images obtained from spherical and hyperbolic convex mirrors it was observed that a large area is occupied by the robot itself thus reducing the total amount of valuable and useful information on the image. It was also observed that by convoluting the whole robot image some distortion is created to the game balls near the image centre becoming a drop shape ball. This severe distortion affects the algorithms that detect the game ball by its circular shape. Fig. 7 shows an example of an image strongly convoluted in the centre (left) and with a small convolution (right).
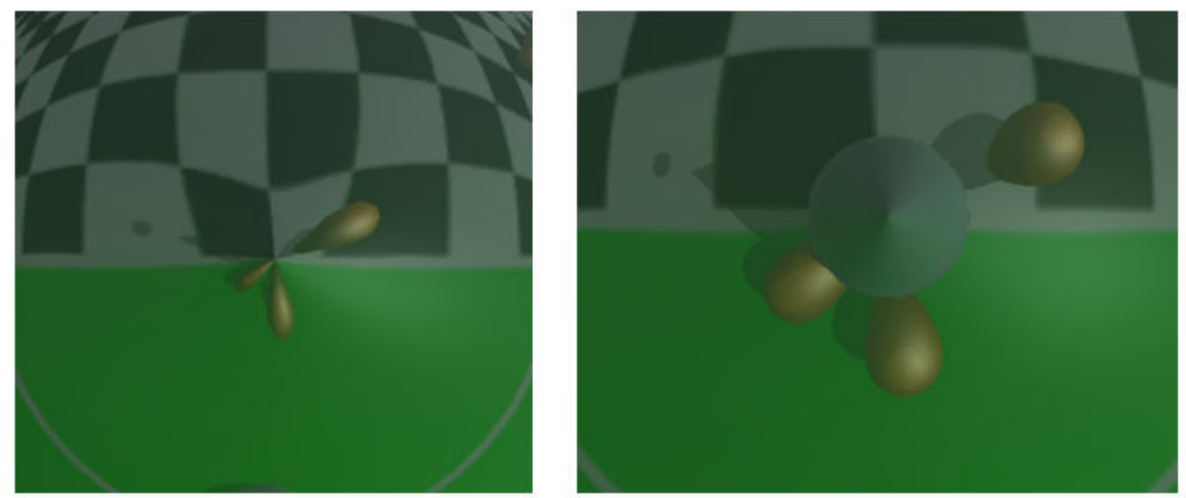

Fig. 7 - Distortion of soccer balls produced by a strong image convolution (left) and a small image convolution (right)

Another important aspect is the robot control and how well defined and visible in the captured image are the objects around it, such as the other robots and the game ball. A three meter radius was chosen to be a good distance that should be well defined in the captured image to allow the robot to have a good performance in the control algorithms when moving at very high speeds. This helps avoiding robot collisions and allows an improved representation of the soccer ball, simplifying the tackle and dribble. To attain this it is necessary to provide the convex mirror with a curvature that can create an image augmentation or zoom in the area up to the defined three meter radius. Beyond that the reflected image can be linear, i.e., a straight line in the mirror line definition will reflect the image proportionally.

The previous description shows how the mirror line definition was chosen and how the line segments define the areas of reflection. In brief, a curve or spline starting from the mirror centre will reduce the size of the robot on the image carrying on to perform the augmentation area. A straight line is used in the edge side to obtain a proportional image. Fig. 8 shows the final version of the developed mirror. 


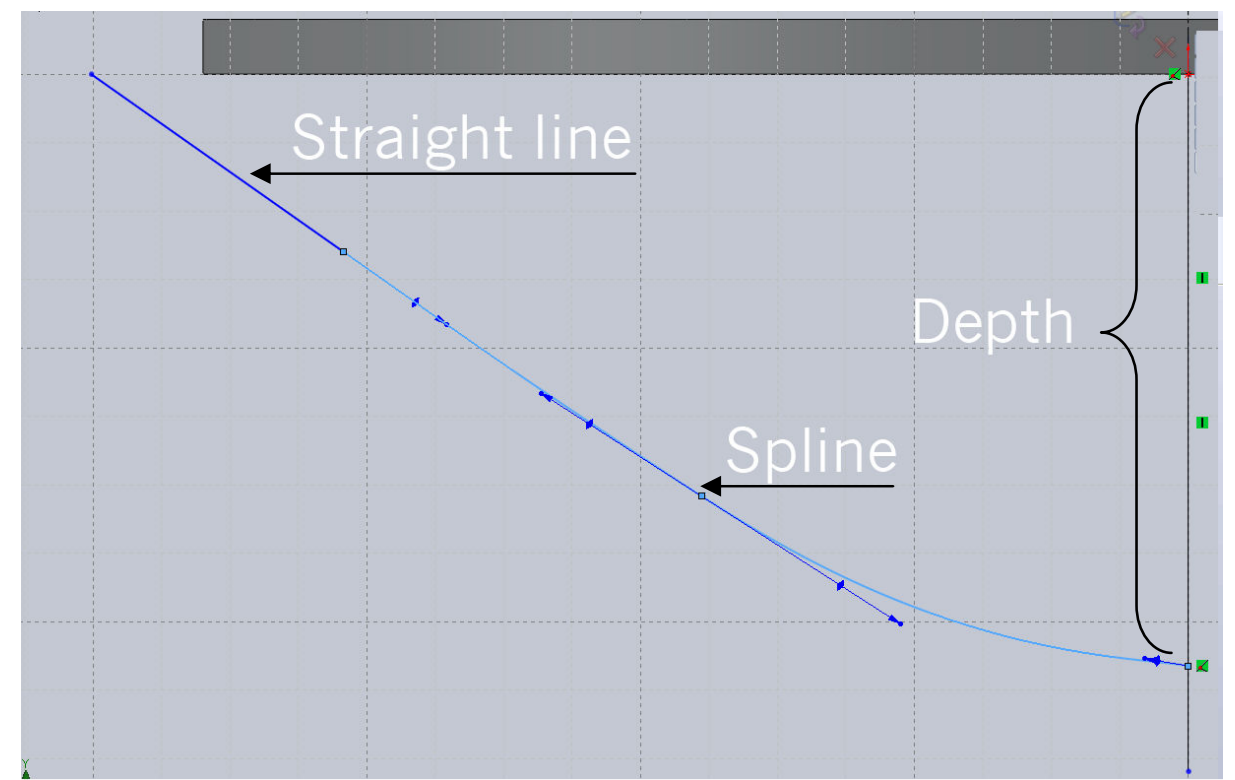

Fig. 8 - Final approach of our developed mirror

One important parameter is the convexity or mirror depth (see Depth on Fig. 8). This parameter will define the distance of the robot sight but it must be balanced with other parameters in order to provide a proper image and to avoid major distortions. Some trial-and-error is essential to get the most out of each parameter until the final definition. Table 1 summarizes the final parameters of the developed mirror in our MSL robots. It should also be noted the importance of the lens definition in order to achieve a proper match afterwards when the mirror is machined.

Table 1 - Parameters of the final developed convex multi-part mirror

\begin{tabular}{|l|l|}
\hline \multicolumn{1}{|c|}{ Parameter } & \multicolumn{1}{c|}{ Value } \\
\hline Spline - part 1 - X & 0.00 \\
\hline Spline - part 1 - Y & -21.60746101 \\
\hline Spline - part 1 - angle & $-9.29656199^{\circ}$ \\
\hline Spline - part 2 - X & -17.76355387 \\
\hline Spline - part 2 - Y & -15.42003468 \\
\hline Spline - part 2 - angle & $-32.69814149^{\circ}$ \\
\hline Spline - part 3 - X & -30.87114791 \\
\hline Spline - part 3 - Y & -6.47671516 \\
\hline Spline - part 3 - angle & $-35.08394856^{\circ}$ \\
\hline Straight line length & 11.25296534 \\
\hline Straight line angle & $144.86140003^{\circ}$ \\
\hline
\end{tabular}

\subsection{Machining the mirror}

When a good result was obtained in the simulation, it was necessary to export the data in a format that could be used by a CNC lathe to produce the convex mirror. SolidCAM software package integrates with SolidWorks when it detects its existence during the installation. This software establishes a bridge between the 3D 
models developed by the CAD software and the machining world. It can generate the necessary code to perform the machining and the language chosen was the G-code (language used by the CNC lathe available at the mechanical workshop). Some metals were tested such as stainless steel and aluminium and after polishing to a fine grain size some differences were noticed between the two mirrors. The images from the stainless steel mirror were slightly darker than the ones reflected by the aluminium mirror. The mechanical engineering department suggested the use of brass with a coating of chromium, but that was our decision since the weight of a brass mirror would be equivalent to the stainless steel and they are much heavier than aluminium. In that sense, aluminium was the preferred material to build up the robot mirrors. The final result can be seen in Fig. 9 where the simulated mirror is shown side-by-side with the machined mirror.
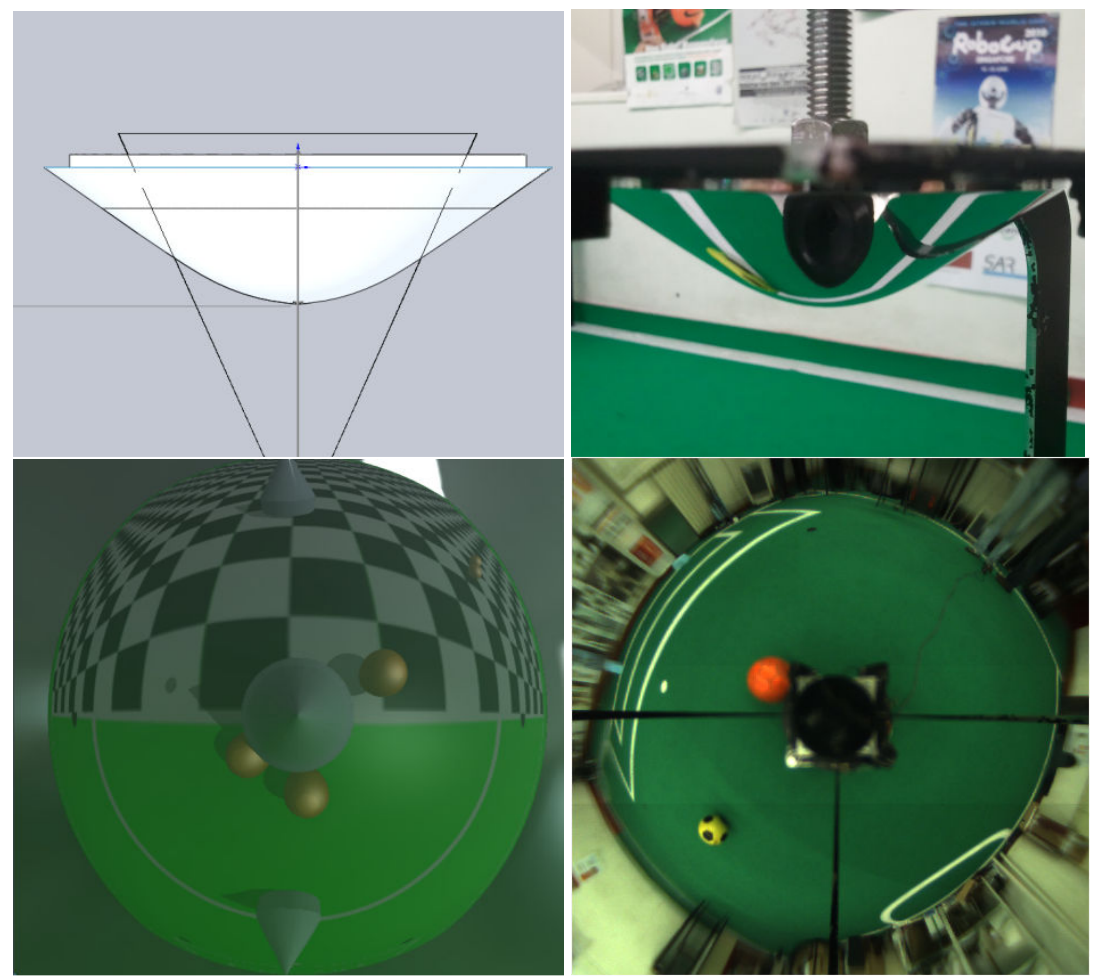

Fig. 9 - Final result of the simulated mirror side-by-side with the machined mirror

\section{Robot head}

The robot head is the part where the camera/lens and the convex mirror are fixed. They define how the image will look like. The distance between the lens and the mirror dictates whether the simulated scenario will match the real scenario. This is where our second approach to improve the efficiency takes place. Commonly, teams define the distance from camera and mirror in order to obtain the whole mirror image. Since the camera sensor is square, a circular image will be seen on the image centre 
and four black corners will fill up the rest. As it was discussed earlier, every pixel counts when processing an image resulting in a loss of image definition due to the resultant lower resolution. To counteract with this, our approach is to approximate the camera with the mirror in order to fill the whole camera sensor with valid image reflected from the convex mirror. The $3 \mathrm{D}$ simulation was already performed taking this into account. It maximises the use of the convex mirror to capture as much as possible the robot surroundings.

Another important aspect of the robot head is how the convex mirror is fixed and attached to the robot allowing visibility and sturdiness. Different methods are used by the teams from acrylic pipe to a fixture of three or four pillars that support a flat top. The first option produces image glare and brightness inconsistencies produced by the round shape acrylic. The second option though produces loss of valid image since the pillars can be seen on the image. To reduce this occlusion, the head was drawn with the pillars being as thin as possible and put in a way that would be far from the mirror reducing the occlusion. Three pillars were used and displaced as a cross to avoid any obstruction or occlusion in the $180^{\circ}$ angle in front of the robot. The developed robot head is shown in Fig. 10.
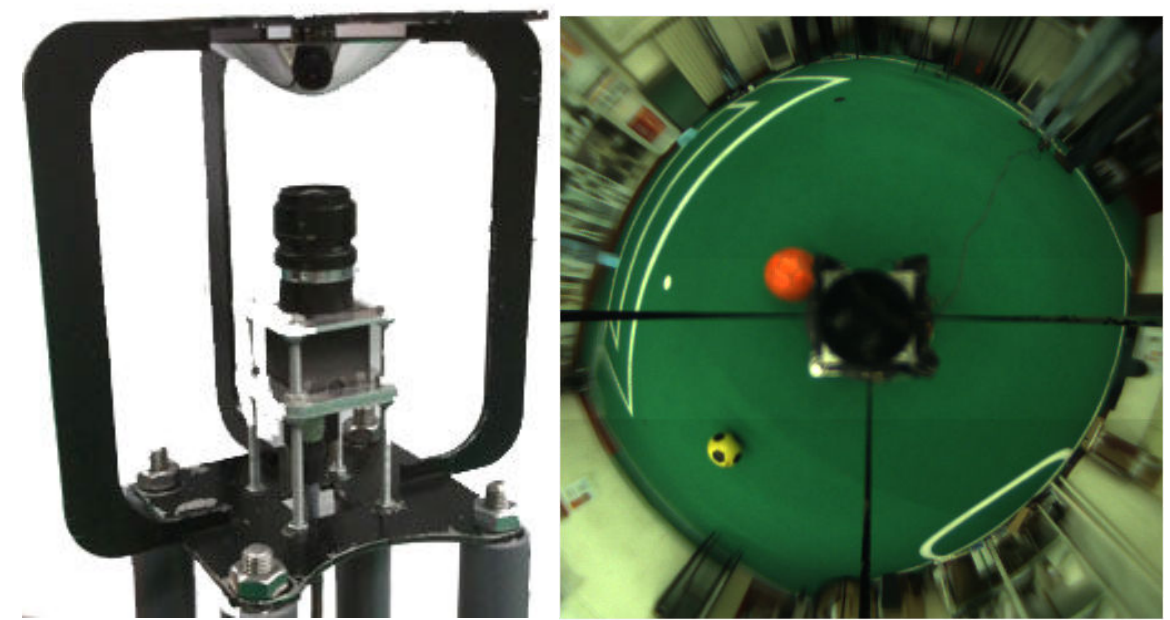

Fig. 10 - Robot head with the thin pillars and corresponding captured image

\section{Conclusions}

Computer 3D modelling and simulation of convex mirrors for applications such as omnidirectional robot vision of MSL robots was successfully attained as it was described in this paper. Two methods were proposed to increase the efficiency of omnidirectional vision systems where all the image information is useful with only a few pixels discarded. Mirror simulation and machining enables an easy and faster production of different curvature shapes for customised purposes increasing specific areas of visibility to the vision processing system. Aluminium has also shown to be a better material for the production of convex mirrors due to the final brighter image reflection and to be a lighter material when compared with stainless steel or brass 
metals. The robot head was developed to reduce the occlusions created with the fixtures that hold the convex mirror to the robot body.

\section{Acknowledgements}

The authors wish to thank the ALGORITMI Research Centre for the opportunity to develop this research, and also wish to thank the department of Mechanical Engineering in the person of Prof. Caetano Monteiro for the use of the $\mathrm{CNC}$ lathe and help on the mechanical development.

\section{References}

1. Bonarini, A., et al. Omnidirectional vision. 2010 2010]; Available from: http://robocup.elet.polimi.it/MRT/OmniVision.html.

2. Azevedo, J.L., et al., CAMBADA'2008: Team Description Paper, in Robocup MSL TDP. 2008, Universidade de Aveiro. p. 9.

3. Lauer, M., S. Lange, and M. Riedmiller, Calculating the Perfect Match: an Efficient and Accurate Approach for Robot Self-Localization, in RoboCup 2005: Robot Soccer World Cup IX. 2006, Springer Berlin / Heidelberg.

4. Winters, N. and J. Santos-victor, Mobile robot navigation using omnidirectional vision, in In Proc. 3rd Irish Machine Vision and Image Processing Conference (IMVIP'99). 1999. p. 151-166.

5. Siemiatkowska, B. and R. Chojecki, Mobile Robot Navigation Based on Omnidirectional Sensor, in Proc. 1st European Conference on Mobile Robots ECMR'03, EURON Conference, Radziejowice 2003. p. 6.

6. Neves, A.J.R., et al., An efficient omnidirectional vision system for soccer robots: From calibration to object detection. Mechatronics.

7. Yi, S. and N. Ahuja, A Novel Omnidirectional Stereo Vision System with a Single Camera in Scene Reconstruction Pose Estimation and Tracking, R. Stolkin, Editor. 2007, I-Tech Education and Publishing. p. 454-466.

8. Marchese, F.M. and D.G. Sorrenti, Omni-directional vision with a multi-part mirror in RoboCup 2000: Robot Soccer World Cup IV, Lecture Notes in Computer Science. 2001. p. 179-188.

9. Gaspar, J.A.d.C.P., Omnidirectional Vision for Mobile Robot Navigation. 2002, IST - Universidade Tecnica de Lisboa. p. 150.

10. Ishiguro, H., Development of Low-Cost Compact Omnidirectional Vision Sensors and their applications, in Panoramic Vision, chapter 3. 1998, Springer. p. 433--439.

11. SolidWorks. Dassault Systèmes - SolidWorks Corp. 2010 [cited 2010; Available from: http://www.solidworks.com/.

12. SolidCAM. The leaders in integrated CAM. 2010 [cited 2010; Available from: http://www.solidcam.com/. 